\title{
Effect of exogenous melatonin and extending the dark period at dusk before the summer solstice on the onset of oestrus in Romney Marsh ewes
}

\author{
M. V. Guerin, A. J. Napier and C. D. Matthews \\ Department of Obstetrics and Gynaecology. The University of Adelaide, The Queen Elizabeth Hospital, \\ Woodville 5011, South Australia
}

\begin{abstract}
Groups of Romney Marsh ewes $(n=24)$ were injected with exogenous melatonin or subjected to extended darkness or a combination of both at dusk from the winter to the summer solstice before being re-exposed to the natural photoperiod at $35^{\circ} \mathrm{S}$. The animals were at all times exposed to natural dawn. The onset of subsequent oestrus was delayed when compared with controls subjected to the natural photoperiod. Either melatonin or darkness or a combination were equally effective at delaying oestrus. The results are consistent with a hypothesis based on an external coincidence model of seasonal breeding involving the coincidence of a light-sensitive phase set close to dusk that, when exposed to light in spring and summer, prepares the reproductive axis to respond correctly to the direct effects of the inductive photoperiod in autumn. The offset of the time of oestrus was not affected by the experimental treatments nor was the timing of the subsequent reproductive season. Since melatonin was as effective as darkness at influencing the assumed photosensitive signal, even in the presence of natural light, melatonin may act through the light reception system.
\end{abstract}

\section{Introduction}

The mechanism of photoperiodic time measurement may involve the presence of an endogenous circannual cycle of reproductive activity that is precisely synchronized to the optimum time of year by the natural change in photoperiod and the interaction of the pineal hormone melatonin (Malpaux ef al., 1989; see Karsch et al., 1991 for review). The exact way in which melatonin interacts with the putative endogenous annual rhythm of reproduction is still unknown. The most widely studied hypothesis is that the differing duration of melatonin secretion provides the important signal through the year (Rollag et al., 1978; Lincoln and Short, 1980; Arendt et al., 1981; Kennaway et al., 1983; Karsch et al., 1986). An alternative hypothesis is that the timing of breeding depends on the coincidence of a circadian phase sensitive to melatonin with endogenous melatonin secretion (Rollag et al., 1978); evidence for this hypothesis has been limited because it is difficult to determine a circadian phase sensitive to melatonin.

The control of melatonin secretion is believed to reside in the spontaneous circadian activity of the suprachiasmatic nucleus (SCN) of the hypothalamus (Moore and Klein, 1974; Lincoln et al., 1985). Thus, melatonin has proved useful as a peripheral marker of changing endogenous (circadian) activity or the 'biological clock' (Illnerova and Vanacek, 1982, 1983; Lewy, 1983). We presented evidence that the usual reported pattern of endogenous melatonin secretion in Romney Marsh

Received 3 August 1993. sheep, which is closely linked to the duration of darkness, may not be fully representative of the SCN signal but reflects only a gated version (by natural photoperiod) of clock activity particularly in the spring and summer. Measurements of melatonin secretion under conditions of acutely extended (to avoid entrainment effects) darkness suggest that there is a period, particularly during the spring and summer, when the natural light of dusk suppresses the expression of melatonin (Matthews et al., 1992).

This induction of SCN activity before dusk in spring and summer, but after dusk in winter, may provide an alternating light-dark signal throughout the year to prepare the reproductive axis during long days to respond directly to autumn. In the current experiment the hypothesis that the time preceding dusk in long days is crucial to the timing of seasonal reproductive activity and is more influential than the autumn period in timing reproductive activity was tested. The experiments were performed at $35^{\circ} \mathrm{S}$. latitude under as natural conditions as possible.

Preliminary reports have appeared in the Abstracts of 25th Annual Meeting of the Society for the Study of Reproduction, and of the 3rd Meeting of the Society for Research on Biological Rhythms.

\section{Materials and Methods}

Before the experiments began, a suitable dose and route of administration of melatonin to maintain blood concentrations Downloaded from Bioscientifica.com at 04/26/2023 11:58:30AM 
at physiological or higher values for $5 \mathrm{~h}$ were determined in a group of age-matched Romney Marsh ewes. Oral, i.m. and s.c. melatonin administration at a range of doses were administered, followed by collection of blood samples at intervals of $30 \mathrm{~min}$ for $5 \mathrm{~h}$. The dose and route selected were $1.0 \mathrm{ml}$ i.m. injection of $3.0 \mathrm{mg}$ melatonin (Sigma Chemical Co., St Louis, $\mathrm{MO}$ ) in $10 \%$ ethanol-90\% peanut oil. The plasma clearance of melatonin injections is shown in the results section.

The experiment was performed on 1.5-year-old Romney Marsh ewes $(n=24)$ at the Waite Agricultural Research Institute, South Australia $\left(35^{\circ} \mathrm{S}\right)$. Sheep were acclimatized for 3 weeks before treatments began at the winter solstice (June 1989); all ewes were initially confirmed to be in oestrus by laparoscopy. Ewes were physically and visibly isolated from rams throughout the entire experiment.

The treatments were conducted in open-sided sheds with good access to natural light at all times of the day and year. Animals were randomly assigned to four groups: (a) control; (b) melatonin treated; (c) afternoon darkness; and (d) afternoon melatonin treatment and darkness. All animals received either a melatonin or control injection each day at $15: 00 \mathrm{~h}$ during the treatment phase. The groups not treated with melatonin received an injection of $1.0 \mathrm{ml} 10 \%$ ethanol-90\% peanut oil. Groups (a) and (b) received only natural light and were housed in individual pens. Groups (c) and (d) were subjected to early afternoon darkness and were housed in groups of four in three larger pens. Darkness at 15:00 h was achieved by the use of light-proof blinds, which were removed well after natural sunset to allow access to the natural light of dawn. At the completion of the treatment period (the summer solstice), all animals were placed in an open paddock for the remainder of the study. The exogenous melatonin and additional darkness treatments were given daily at $15: 00 \mathrm{~h}$ to ensure coverage of the proposed sensitive phase identified by Matthews et al. (1992) between winter and summer.

Before the experiments began, and at intervals of 1 month until the completion of treatment, blood samples of $10 \mathrm{ml}$ were collected from all sheep at intervals of $30 \mathrm{~min}$ (by syringe and needle) to determine the onset of the endogenous melatonin rise. The actual sampling times varied between groups to ensure the melatonin rise was detected, and collection of blood samples from all animals was carried out within 6 min (maximum) of the stated collection time. Animals receiving exogenous melatonin did not receive a melatonin injection on the sampling night. In October, sampling at intervals of $30 \mathrm{~min}$ was conducted in the morning to determine the offset of high endogenous melatonin concentrations.

At the completion of the treatment period, oestrus was monitored by the collection of blood samples of $10 \mathrm{ml}$ twice a week for the determination of plasma progesterone concentrations. Animals were considered to be in oestrus when progesterone concentrations exhibited a regular pattern of rise and fall. The commencement of oestrus was taken as the date of the first progesterone value recorded above the anoestrous baseline value when part of a recurrent cyclic pattern. The offset of oestrus was taken as the date of the last progesterone value above baseline at the completion of the cyclic progesterone pattern.

All times were corrected for local summer time and therefore based on the natural photoperiod. After oestrus all animals were maintained in natural conditions, and similar measurements were made to determine the time of onset of the next (1991) oestrus.

\section{Melatonin measurement}

Melatonin was assayed in duplicate with $500 \mu \mathrm{l}$ plasma aliquots using the extracted radioimmunoassay method of Kennaway et al. (1982), with modifications by Earl et al. (1985). The intra-assay coefficients of variation over repeated measurements at concentrations of $250 \mathrm{pmol} \mathrm{I}^{-1}$ and $1200 \mathrm{pmol} \mathrm{l^{-1 }}$ were $10.6 \%(n=19)$ and $6.8 \%(n=19)$, respectively. The interassay coefficients of variation over the course of the assays at similar concentrations were $12.5 \%(n=18)$ and $9.6 \%$ $(n=18)$, respectively. The sensitivity of each assay was calculated as $95 \%$ of the zero standard and the mean and SEM of all assays was $74.2 \mathrm{pmol}^{-1}\left( \pm 19.1 \mathrm{pmol} \mathrm{l}^{-1}\right)$. Samples from each month's collection were normally measured over four assays, with all samples from one treatment group in the one assay. The onset (and offset) of the melatonin rise was defined as the initial sample with detectable (or absent) melatonin above (or below) the assay sensitivity, provided subsequent samples sustained the rise (or absence).

\section{Progesterone measurement}

Progesterone was assayed using the direct ${ }^{125} \mathrm{I}$ radioimmunoassay of Farmos Diagnostics (Turku), and charcoalfiltered sheep plasma was used as a quality control. The recovery data on spiked samples of $4.5 \mathrm{nmol}^{-1}, 9.1 \mathrm{nmol} \mathrm{l}^{-1}$ and $18.2 \mathrm{nmol} \mathrm{l}^{-1}$ were $100 \%, 99.0 \%$ and $97.3 \%$, respectively. The intra-assay coefficients of variation over repeated measurements at concentrations of $6.4 \mathrm{nmol}^{-1}$ and $30 \mathrm{nmol} \mathrm{l}^{-1}$ were $7.4 \%$ and $9.5 \%(n=23)$, respectively. The interassay coefficients of variation over the course of the assays at similar concentrations were $16.6 \%$ and $10.0 \%(n=23)$, respectively. The sensitivity of each assay was calculated as the smallest detectable concentration, equivalent to twice the SD of the zero binding value (approximately $0.5 \mathrm{nmol} \mathrm{l}^{-1}$ ) as per the manufacturer's protocol.

\section{Statistical analyses}

The rise (and fall) of melatonin was tested against the time of dark onset (or offset) by using one-sample Student's $t$ tests on the resultant intervals between the rise (or fall) of melatonin and the time of dark onset (or offset). The onset of oestrus between groups in 1989 and 1990 was tested using unpaired $t$ tests on the day of onset of oestrus; and day 1 was the summer solstice of the previous year (21 December). Similarly, the day for the completion of oestrus (1990) was calculated from the summer solstice set as day 1 . The duration of oestrus was also tested using unpaired $t$ tests of the number of days in oestrus for each animal.

\section{Results}

Four hours after i.m. injection of $3.0 \mathrm{mg}$ melatonin in $1.0 \mathrm{mI}$ $10 \%$ ethanol- $-90 \%$ peanut oil, plasma melatonin concentrations Downloaded from Bioscientifica.com at 04/26/2023 11:58:30AM 


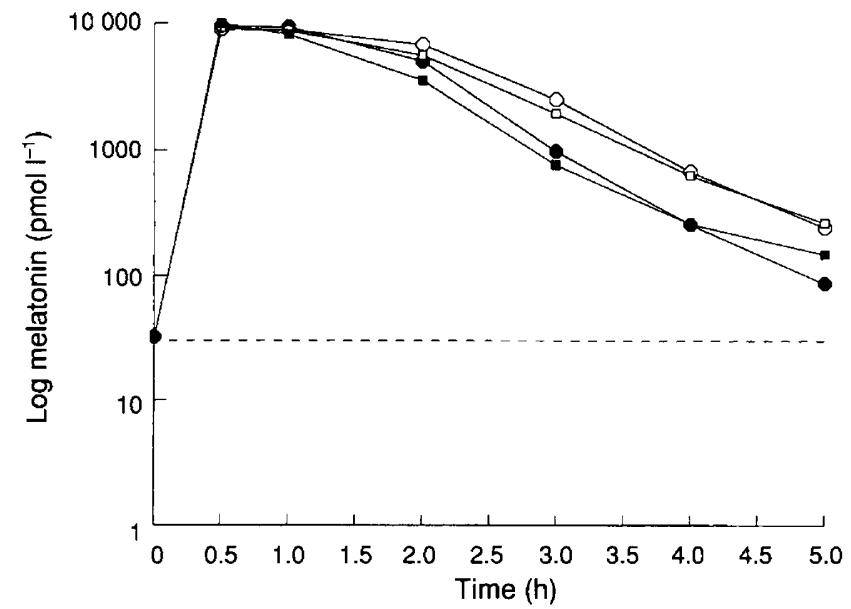

Fig. 1. The individual clearance of melatonin (shown as the log value in pmol $1^{-1}$ ) in the plasma of four ewes following the injection of $3 \mathrm{mg}$ melatonin i.m. in $1 \mathrm{ml} \mathrm{10 \%}$ ethanol-90\% peanut oil vehicle. Time (h) from the time the injection was administered is shown, where $\mathrm{oh}$ was $2 \mathrm{~h}$ after sunrise and the melatonin value at this point was below the assay sensitivity $\left(32 \mathrm{pmol} \mathrm{I}^{-1}\right)$. The dashed line indicates the assay sensitivity level.

of all animals were $250 \mathrm{pmol}^{-1}$ or greater, and after $5 \mathrm{~h}$ melatonin concentrations were $87 \mathrm{pmol}^{-1}$ or greater (Fig. 1). At the completion of the experimental treatment period on 21 December (the summer solstice), natural sunset time is 19:29 h ( $4.5 \mathrm{~h}$ after injection time), an injection of $3 \mathrm{mg}$ melatonin would therefore be expected to maintain at least the physiological value from $15: 00 \mathrm{~h}$ to the onset of natural darkness throughout the treatment period.

The results of collection of blood samples at intervals of 1 month for the monitoring of the onset of the melatonin rise are illustrated (Fig. 2). Contamination of samples in the June and July samples precluded reporting of these results. The contamination was assumed to result from the handling of the concentrated melatonin solution for injections, and all handling and cleaning procedures were modified to avoid contamination for the remainder of the experiment.

Groups (a) and (b) receiving only natural light displayed a melatonin rise that occurred later than sunset $(P<0.05)$ at all months except for group (b) in October, which displayed a melatonin rise not different from that at the time of sunset. The time of the fall in melatonin, determined only in October, was later than sunrise for group (b) $(P<0.01)$, but not different from the time of sunrise in the other groups. Groups (c) and (d) were subjected to darkness from 15:00 h but natural sunrise during the treatment period. Both groups displayed a melatonin rise at about 15:00 h in October and group (c) displayed a melatonin rise at about 15:00 h in December. At all other times of measurement, the melatonin rise occurred later than $15: 00 \mathrm{~h}$ $(P<0.01)$.

The onset, offset and duration of oestrus after the completion of treatments and the onset of oestrus in the following year for all sheep is shown (Table 1). The onset of oestrus in the control group began on average 102.6 days after the summer solstice (range, 84-112 days). All other groups displayed delayed onset of oestrus after the summer solstice relative to controls $(P<0.001)$. There was no difference between groups in the timing of the cessation of oestrus; hence, oestrus in the control group lasted longer $(P<0.01$, groups $(b)$ and $(\mathrm{d}) ; P<0.05$, group $(\mathrm{c}))$. There was no difference between groups in the onset of oestrus in the subsequent year (1991).

\section{Discussion}

Administration of darkness or exogenous melatonin or both factors at dusk between the winter and summer solstices was effective in delaying oestrus compared with controls submitted to the natural photoperiod. Since all groups of sheep were exposed to the natural photoperiod from the summer solstice, it seems that the observed differences in oestrus timing were due to the treatment programmes. The effect achieved can be explained by the treatments interfering with the reception of a light-based signal, probably (though not studied here) coincident with the commencement of SCN activity. The results are consistent with the findings of a number of studies (Robinson et al, 1985; Malpaux et al., 1989) that a long-day signal is necessary to time breeding.

Extending darkness or providing exogenous melatonin at dusk has in previous studies served only to advance the breeding time when given close to the natural time of breeding (Kennaway et al., 1982; Nett and Niswender, 1982; Arendt et al., 1983). Such results are consistent with darkness or melatonin mimicking the autumn (or direct) effects on reproduction by simply extending the duration of melatonin secretion. The current findings that similarly timed treatments at dusk delayed oestrus has demonstrated the need for a preliminary long-day signal to sensitize the reproductive system for a response to the autumn photoperiod to occur.

The finding that melatonin injections (group (b)) are as effective as darkness (group (c)) at delaying oestrus suggests that the assumed photosensitive phase to light 'interprets' melatonin as darkness. Presumably, this blocking effect during the time of long photoperiod is similar to the advancement of oestrus by melatonin administration after the summer solstice (Kennaway et al., 1982; Nett and Niswender, 1982; Arendt et al., 1983). Exactly how melatonin equates to darkness under these circumstances remains unknown. All experimental groups presumably had melatonin present during the time of the assumed photosensitive phase (groups (c) and (d), endogenously owing to the additional darkness, and group (b), exogenously) and therefore it is not possible to distinguish between the effect of darkness and melatonin.

Measurements of endogenous melatonin concentrations during the experiment (under treatment conditions) confirmed that the onset of melatonin secretion with additional darkness at dusk (groups (c) and (d)) occurred earlier than in controls. It is not clear whether this reflected a true phase advance of the onset oscillator (single or multiple) or whether true clock activity was simply unmasked. An advanced rise in the endogenous melatonin concentration was not seen in group (b) animals that, although subjected to regular melatonin injections before natural dusk, were housed in natural conditions and therefore had access to natural light. There was therefore little evidence that melatonin phase-advanced circadian activity when administered in the presence of natural dusk light. Downloaded from Bioscientifica.com at 04/26/2023 11:58:30AM 
(a)

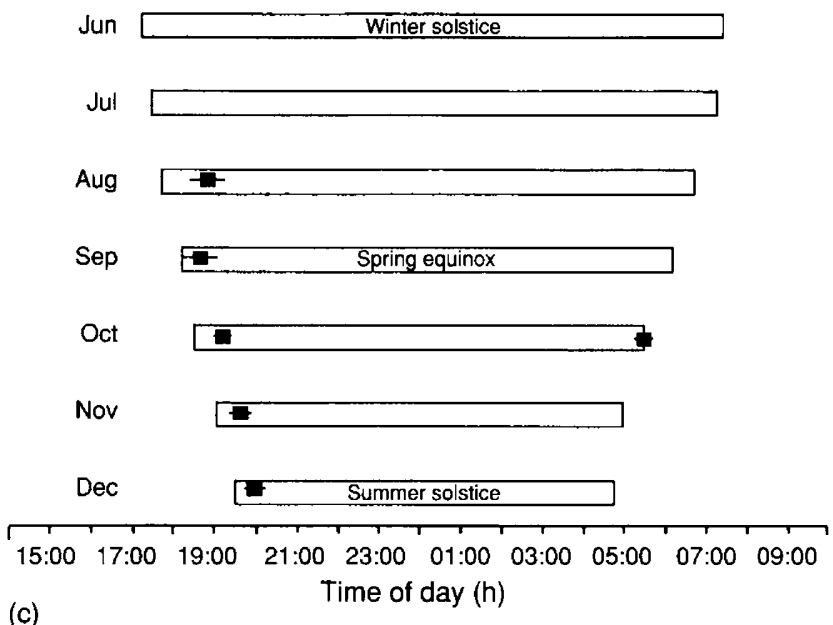

(c)

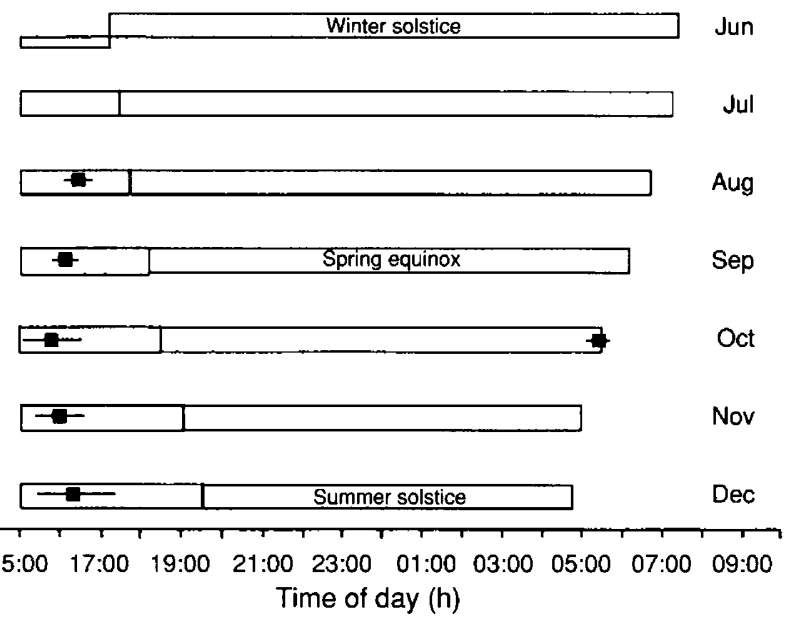

(b)

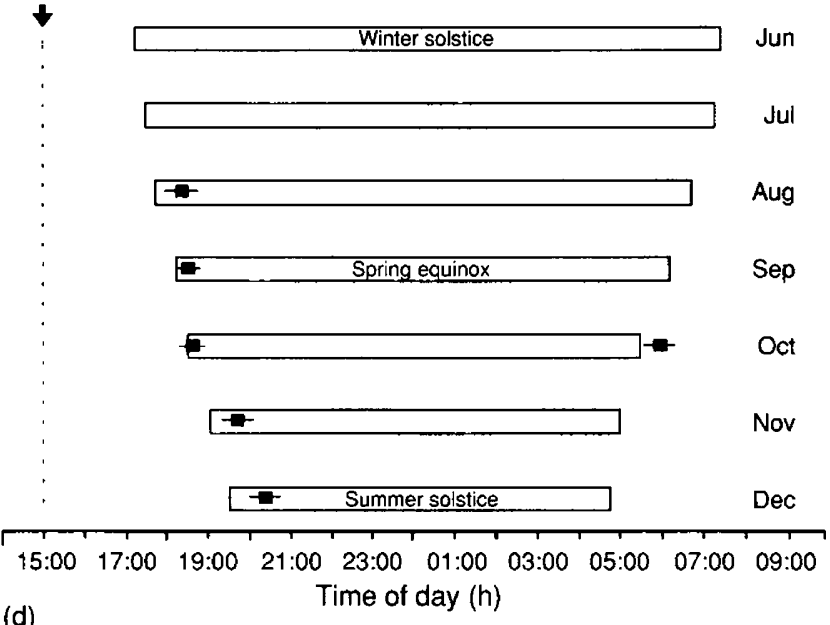

(d)

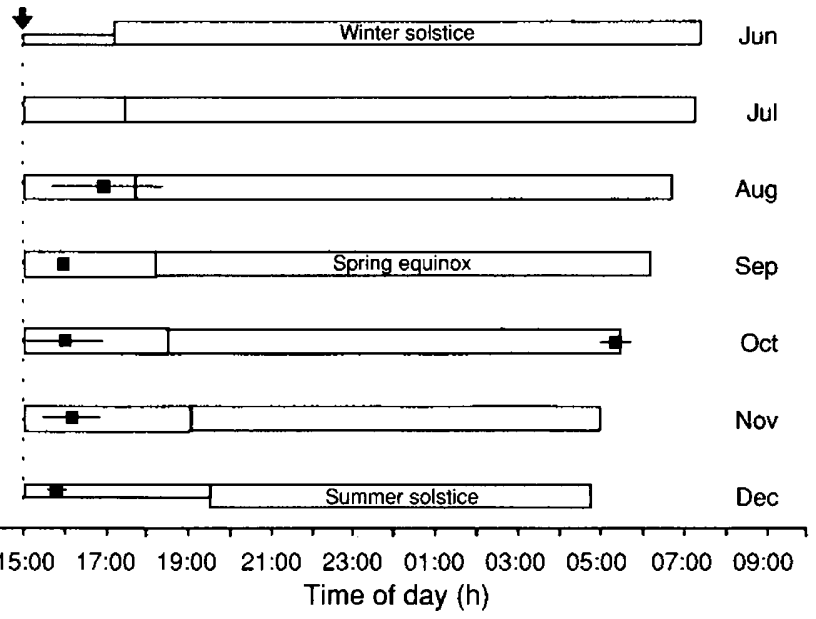

Fig. 2. Onset and offset times of melatonin secretion ( \pm SEM) for the four treatment groups throughout the treatment period: (a) control; (b) melatonin treatment; (c) aftemoon darkness; and (d) aftemoon melatonin treatment and darkness. The open boxes represent the period of darkness (at the times of collection of monthly samples from June to December) and for groups (c) and (d), where there was additional darkness, the natural sunset time is marked by the vertical line through the open bars. The dashed lines (arrowed) illustrate the time of daily melatonin administration. Treatments began on 21 June and finished on 21 December. The solid squares $(\square)$ show the time of endogenous melatonin onset (for August to December) and offset (October), with the SEM indicated for each measurement. The winter and summer solstices and the spring equinox are noted. Clock times have been adjusted for periods of daylight saving.

However, no definite statements can be made concerning the true onset position of SCN activity, since measurements of melatonin concentrations in acutely extended darkness were not performed in this particular study.

After the summer solstice, all sheep were exposed to the natural photoperiod and the offset of reproductive activity was not different in any group. Thus, the overall duration of oestrus was shorter than normal in all three groups that commenced activity later than the controls. O'Callaghan et al. (1992) suggested that genetic differences may explain differences in duration of endogenous reproductive activity between breeds. The results of this study do not suggest a defined duration to the breeding season that can simply be phase-shifted by manipulating the photoperiod before the summer solstice. Rather, they suggest a common response to some environmental event, possibly photoperiod, to terminate the breeding season. Since breeding extends past the winter solstice, the termination signal may involve a response to increasing day length. Although the increase in photoperiod (at the latitude of the experiment) is about $30 \mathrm{~min}$ when breeding ceases, there is evidence (C. D. Matthews, M. V. Guerin and A. J. Napier, unpublished) that at this time light is already impinging on SCN activity at the onset of dusk.

The onset of the following oestrous season (1991) was normal in all groups, although about 2 weeks later than in 1990. Thus, the experimental treatment did not influence the following breeding season. This has been a common finding in a number of studies (Stables et al., 1992).

The protocol of this study was designed to give access to natural dawn on all days, which gradually becomes earlier from spring to summer. The tau (the period of the endogenous circadian activity) of the Romney Marsh sheep appears to be $>24 \mathrm{~h}$ (Matthews et al., 1992) and so the time of dawn is likely to be an important entraining influence, tending to Downloaded from Bioscientifica.com at 04/26/2023 11:58:30AM 
Table 1. The onset, offset and duration of oestrus following the completion of treatment (1990) and the onset of oestrus in the subsequent year (1991) for all sheep, with group means and SEs, where the number of days is the number of days from the summer solstice of the previous year (21 December)

\begin{tabular}{|c|c|c|c|c|}
\hline Group & $\begin{array}{l}1990 \text { Oestrus onset } \\
\text { (number of days) }\end{array}$ & $\begin{array}{l}\text { Anoestrus onset } \\
\text { (number of days) }\end{array}$ & $\begin{array}{l}\text { Duration of oestrus } \\
\text { (days) }\end{array}$ & $\begin{array}{l}1991 \text { Oestrus onset } \\
\text { (number of days) }\end{array}$ \\
\hline \multicolumn{5}{|l|}{ Control } \\
\hline$I$ & 98 & 236 & 138 & 76 \\
\hline 2 & 84 & 222 & 138 & 69 \\
\hline 3 & 105 & 215 & 110 & 83 \\
\hline $4^{a}$ & - & - & - & - \\
\hline 5 & 112 & 222 & 110 & 80 \\
\hline 6 & 109 & 208 & 99 & 90 \\
\hline Mean $( \pm \mathrm{SE})$ & $102.6(10.0)$ & $220.6 \quad(9.3)$ & $119(16.0)$ & $79.6 \quad(7.0)$ \\
\hline \multicolumn{5}{|l|}{ Group b } \\
\hline 7 & 117 & 215 & 98 & 76 \\
\hline 8 & 165 & 208 & 43 & 97 \\
\hline 9 & 134 & 208 & 74 & 76 \\
\hline 10 & 144 & 222 & 78 & 76 \\
\hline 11 & 130 & 228 & 98 & 87 \\
\hline 12 & 152 & 236 & 84 & 111 \\
\hline Mean $( \pm \mathrm{SE})$ & $140.3(15.5)$ & $219.5(10.3)$ & $79.2(18.6)$ & $87.2(13.2)$ \\
\hline \multicolumn{5}{|l|}{ Group c } \\
\hline 13 & 130 & 222 & 92 & 76 \\
\hline 14 & 130 & 215 & 85 & 83 \\
\hline 15 & 130 & 215 & 85 & 73 \\
\hline 16 & 126 & 180 & 54 & 94 \\
\hline 17 & 140 & 236 & 96 & 108 \\
\hline 18 & 109 & 222 & 113 & - \\
\hline Mean $( \pm \mathrm{SE})$ & $127.5 \quad(9.3)$ & $215(17.1)$ & $87.5(17.7)$ & $86.8(12.8)$ \\
\hline \multicolumn{5}{|l|}{ Group d } \\
\hline 19 & 133 & 243 & 110 & 87 \\
\hline 20 & 137 & 215 & 78 & 73 \\
\hline 21 & 140 & 222 & 82 & 87 \\
\hline 22 & 147 & 222 & 75 & 108 \\
\hline $23^{2}$ & - & - & - & - \\
\hline 24 & 105 & 187 & 82 & 73 \\
\hline Mean $( \pm \mathrm{SE})$ & $132.4(14.4)$ & $217.8(18.0)$ & $85.4(12.6)$ & $85.6(12.8)$ \\
\hline
\end{tabular}

${ }^{a}$ Animals 4 and 23 died of natural causes before the commencement of oestrus.

phase-advance circadian activity in lengthening days according to phase response curve principles (Lewy, 1983) until the dusk light becomes influential and stabilizes SCN activity across the short night. The appreciation of natural dawn may be very important if a circadian element is involved in the timing of reproduction, since the offset of endogenous circadian activity would always tend to be delayed by the unnatural (delayed) time of dawn in 'solstice-hold' experiments (Robinson and Karsch, 1984; Robinson et al., 1985). The effect of such a dawn delay would probably alter the time of onset of melatonin secretion and thus its interaction with the light of dusk. Evidence for this delay of whole SCN activity in winter compared with summer has been reported in humans (Illnerova et al., 1985).
Currently, our working hypothesis is based on the external coincidence model of Pittendrigh and Minis (1964). The crucial element of this model is a photosensitive phase to light set close to dusk, which, if illuminated in the long summer photoperiod, sensitizes the reproductive axis so that the shortening photoperiod of autumn is able to induce reproductive activity directly. This photosensitive phase is phase-locked with the commencement of endogenous circadian $\mathrm{SCN}$ activity or 'clock'. The clock itself is responsive to photoperiodic changes that effectively phase-advance in the summer and phase-delay in the winter. The underlying mechanism of this phase shift is the physiology of the phase response curve and the most important influence in our model is the time of dawn, since the tau of the Romney Marsh sheep is $>24 \mathrm{~h}$. Thus, 
partly as a result of phase advances of the clock and partly because of the later dusk of summer, the photosensitive phase, being phased-locked with clock activity, is exposed to the light of dusk in the long photoperiod. After the summer solstice the clock tends to delay and, together with an earlier dusk, this precludes dusk light from the photosensitive phase and induces reproductive activity. This study has confirmed that the interruption of a photosensitive phase at dusk between winter and summer by darkness or melatonin or both factors delays the timing of the subsequent autumn oestrus. The hypotheses should be further tested by experiments designed to allow phase changes of the clock to avoid reception of the light signal or to provide a long-day signal earlier than normal.

The authors would like to acknowledge the help of S. Walker (for laparoscope examinations), J. Wang (for statistical advice) and staff at the Waite Institute for assistance in conducting the experiment. D. Kennaway is acknowledged for the supply of melatonin antibody and advice on melatonin assay methodology.

\section{References}

Arendt J, Symons AM and Laud C (1981) Pineal function in the sheep evidence for a possible mechanism mediating seasonal reproductive activity Experientia 37 584-586

Arendt J, Symons AM, Laud CA and Pryde SG (1983) Melatonin can induce early onset of the breeding season in ewes Journal of Endocrinology 97 395-400

Earl CR, D'Occhio MJ, Kennaway DJ and Seamark RF (1985) Serum melatonin profiles and endocrine responses of ewes exposed to a pulse of light late in the dark phase Endocrinology 117 226-230

Illnerova $\mathrm{H}$ and Vanacek J (1982) Complex control of the circadian rhythm in $\mathrm{N}$-acetyl transferase activity in the rat pineal gland. In Vertebrate Circadian Systems, pp 285-296 Eds J Aschoff, S Daan and G Groos. Springer Verlag, Berlin

Illnerova $\mathrm{H}$ and Vanacek J (1983) Extension of the rat pineal $N$-acetyl transferase rhythm in continuous darkness and on short photoperiod Brain Research 261 176-179

Illnerova H, Zvolsky P and Vanacek J (1985) The circadian rhythm in plasma melatonin concentration of the urbanized man: the effect of summer and winter time Brain Research 328 186-189

Karsch FJ, Bittman EL, Robinson JE, Yellon SM, Wayne NL, Olster DH and Kaynard AH (1986) Melatonin and photorefractoriness. Loss of response to the melatonin signal leads to seasonal reproductive transitions in the ewe Biology of Reproduction 34 265-274
Karsch FJ, Woodfill CJI, Malpaux B, Robinson JE and Wayne NL (1991) Melatonin and mammalian photoperiodism. Synchronisation of annual reproductive cycles. In Suprachiasmatic Nucleus, pp 217-232 Eds DC Klein, RY Moore and SM Reppert. Oxford University Press, New York

Kennaway DJ, Gilmore TA and Seamark RF (1982) Effect of melatonin feeding on serum prolactin and gonadotrophin levels and the onset of seasonal oestrus cyclicity in sheep Endocrinology 110 2186-2188

Kennaway DJ, Sanford LM, Godfrey B and Friesen HG (1983) Patterns of progesterone, melatonin and prolactin secretion in ewes maintained in four different photoperiods Journal of Endocrinology 97 229-242

Lewy AJ (1983) Biochemistry and regulation of mammalian melatonin production. In The Pineal Gland, pp 77-128 Ed. R Relkin. Elsevier Biomedical, New York

Lincoln GA and Short RV (1980) Seasonal breeding nature's contraceptive Recent Progress in Hormone Research 36 1-52

Lincoln DW, Fraser HM, Lincoln GA, Martin GB and McNeilly AS (1985) Hypothalamic pulse generators Recent Progress in Hormone Research 41 369-419

Malpaux B, Robinson JE, Wayne NL and Karsch FJ (1989) Regulation of the onset of the breeding season of the ewe. Importance of long days and of an endogenous reproductive rhythm Endocrinology 122 269-278

Matthews CD, Seamark RF and Guerin MV (1992) Plasma melatonin profiles of Romney Marsh sheep in natural photoperiod and in acutely extended darkness Journal of Reproduction and Fertility 95 869-875

Moore RY and Klein DC (1974) Visual pathways and the central neural control of a circadium rhythm in pineal serotonin $N$-acetyl transferase activity Brain Research 71 17-33

Nett TM and Niswender GD (1982) Influence of exogenous melatonin on seasonality of reproduction in sheep Theriogenology 17 645-653

O'Callaghan D, Karsch FJ, Boland MP, Hanrahan JP and Roche JF (1992) Variation in the timing of the reproductive season among breeds of sheep in relation to differences in photoperiodic synchronisation of an endogenous rhythm Journal of Reproduction and Fertility 96 443-452

Pittendrigh CS and Minis DH (1964) The entrainment of circadian oscillation by light and their role as photoperiodic clocks American Naturalist 98 261-294

Robinson JE and Karsch FJ (1984) Refractoriness to inductive day lengths terminates the breeding season of the Suffolk ewe Biology of Reproduction $\mathbf{3 1}$ 656-663

Robinson JE, Wayne NL and Karsch FJ (1985) Refractoriness to inhibitory daylength initiates the breeding season of the Suffolk ewe Biology of Reproduction 32 1024-1030

Rollag MD, O'Callaghan PL and Niswender GD (1978) Serum melatonin concentrations during different stages of the annual reproductive cycle in ewes Biology of Reproduction 18 279-285

Stables LD, McPhee S, Kennaway DJ and Williams AH (1992) The influence of exogenous melatonin on the seasonal patterns of ovulation and oestrus in sheep Animal Reproduction Science 30 185-224. 